\title{
EVALUACIÓN DE LA GESTIÓN DE SUSTENTABILIDAD Y SEGURIDAD EN CONSTRUCCIONES DE VIVIENDA EN MÉXICO
}

\author{
Evaluation of sustainable management and safety in \\ housing construction in Mexico
}

\section{EPISTEMUS}

ISSN: 2007-8196 (electrónico)

ISSN: 2007-4530 (impresa)

Marco Antonio Ramos Corella 1

Salvador García Rodríguez ${ }^{2}$

Jesús Quintana Pacheco ${ }^{3}$

Arturo Ojeda De La Cruz 4

Ana Cecilia Borbón Almada 5

Recibido: 19 de septiembre de 2015

Aceptado: 15 de marzo de 2016

Autor de Correspondencia:

Dr. Marco Antonio Ramos Corella

Correo: marco.ramos.corella@gmail.com

\section{Resumen}

Se presentan los resultados de la aplicación de un modelo para determinar el nivel de gestión de sustentabilidad y seguridad en construcciones de vivienda en México. Esta evaluación se determina mediante la aplicación de criterios y parámetros con los cuales se miden el grado de compromiso de la gestión del proyecto con el medio ambiente y la seguridad de los involucrados en él desde la etapa de diseño hasta la demolición o desmontaje final. Los criterios utilizados en este modelo tienen dos funciones: medir lo realizado hasta el momento e indicar qué debe hacerse para corregir las debilidades encontradas. Además, pueden utilizarse como pautas a seguir para planear proyectos futuros y evitar problemas de gestión.

Palabras clave: seguridad ocupacional, sustentabilidad, construcción sostenible.

\begin{abstract}
In this paper are shown the results of a model's implementation to determine the level of sustainable and occupational management for constructions of houses in Mexico. This assessment is determined by the application of criteria and parameters to test the commitment level of the project managers with sustainability and occupational safety and health of all the people involved in the project, since the design phase to the construction's demolition. There are two functions of these criteria: the first is to measure what has been done to a single moment in the projects life, and the second one is to point out what should be done to correct the weaknesses found. Also these criteria and parameters can be used to plan new projects and improve the construction management.
\end{abstract}

Keywords: occupational safety, sustainability, sustainable construction.

1 Depto. de Ingeniería Civil y Minas, Universidad de Sonora / Correo: marco.ramos@dicym.uson.mx 2 Instituto Tecnológico y de Estudios Superiores de Monterrey / Correo: sgr@itesm.mx

3 Depto. de Ingeniería Civil y Minas, Universidad de Sonora / Correo: quintana@dicym.uson.mx

4 Depto. de Ingeniería Civil y Minas, Universidad de Sonora / Correo: ojeda@dicym.uson.mx

5 Depto. de Ingeniería Civil y Minas, Universidad de Sonora / Correo: acborbon@dicym.uson.mx 


\section{INTRODUCCIÓN}

Según el Programa de las Naciones Unidas Para el Medio Ambiente (UNEP) [1], a nivel mundial, los edificios son responsables del $40 \%$ del consumo anual de energía y hasta un $30 \%$ de todos los gases de efecto invernadero (GEI) relacionados con la energía; además, menciona que el sector de la construcción es responsable de un tercio del consumo de recursos de la humanidad, del $12 \%$ del consumo total de agua dulce, y produce hasta un $40 \%$ de los residuos sólidos.

En el caso de México, el sector vivienda consume alrededor del $19 \%$ de la energía que produce el país [2] y es responsable de aproximadamente un $7 \%$ de las emisiones GEI, representando a nivel nacional $49 \mathrm{MTCO}_{2}$ por año [3]. Además, la generación anual estimada de residuos originados por la construcción de vivienda para el año 2011 fue $86.7 \mathrm{~kg}$ por metro cuadrado construido en desarrollos habitacionales, y el total del sector de la construcción fue de $114 \mathrm{~kg}$ por metro cuadrado, lo que equivale a una generación diaria de 16,659.28 toneladas [4].

Lo anterior nos muestra que el sistema económico actual usa recursos de la naturaleza y vuelve a ésta desechos que la dañan de forma irreparable, constituyendo un desarrollo lineal y finito. La construcción sostenible, como parte del desarrollo sostenible, tiene por objetivo pasar de este proceso de construcción lineal y finito a uno cíclico e infinito integrado en la naturaleza, en el cual no existan los conceptos de residuo ni de daño ambiental, sino que los materiales puedan ser transformados y permanezcan [5], lo que permitirá seguir evolucionando para satisfacer las necesidades humanas.

\section{Sustentabilidad en los}

\section{hogares, una inversión rentable}

En México, el número de hogares incrementó 1.1\% entre los años 2010 y 2012, pasó de 29'556,772 a 31'359,562 [6] y se estima que, para la tercera década de este siglo, se tendrán cerca de $\mathbf{4 0}$ millones de viviendas, por lo que se calcula que se requerirá la construcción de alrededor de 11 millones de casas nuevas entre hoy y el año 2030 [3]. Tomando en cuenta la gran demanda existente de vivienda, y que una vivienda verde ahorra al menos $30 \%$ de luz, $50 \%$ de gas y $60 \%$ de agua sin modificar sustancialmente la inversión [7], se vuelve muy atractiva la construcción sostenible y el desarrollo sustentable se convierte en "el camino para alcanzar una economía caracterizada por la prosperidad, equidad y un ambiente saludable $y$, es el marco en el que se integran los objetivos económicos, sociales y ambientales que mejoran las perspectivas de las aspiraciones de una sociedad" [8].

La construcción de edificios sustentables implica varios aspectos, entre ellos, que tengan un desempeño eficiente en el consumo de agua y energía; se proteja el sitio de la obra y aprovechen correctamente la infraestructura existente y sus condiciones para minimizar el uso de transporte privado; se minimice el desperdicio de materiales y se tome en cuenta el impacto causado por su extracción y producción; entre otras estrategias [9]. Es decir, si nos proponemos aplicar medidas de construcción sostenible, "es fundamental adoptar una visión integrada, desde la extracción de las materias primas hasta la gestión de sus residuos una vez derribada la obra" [10].

\section{Fuerza de trabajo propensa a accidentes}

El sector de la construcción emplea en promedio más del $10 \%$ de la fuerza laboral [1] y, en la etapa de edificación, es el sector de la economía que absorbe la mayor cantidad de mano de obra no cualificada del país [4], lo cual indudablemente incide en la siniestralidad. Al utilizar esta mano de obra poco cualificada, aunado a que los empleos son eventuales y las posibilidades de promoción son escasas, disminuye notablemente la calidad y aumentan los riesgos por inseguridad. Y, como menciona Solís [11]:"La seguridad y la salud de los trabajadores de la construcción se insertan dentro de un esquema cambiante, en un ambiente donde el sistema de producción se encuentra en fase de implementación, sin llegar a ser estable, lo cual incide en la gestión de la calidad y productividad de los procesos". Es por ello que, con esta escasa formación, existe poca cultura preventiva.

Desde el enfoque de salud ocupacional, la construcción se encuentra dentro de los primeros lugares a nivel mundial, entre aquéllas que tienen un mayor número de accidentes fatales y no fatales [12], por ejemplo, en los Estados Unidos 4,405 obreros de la construcción murieron durante la jornada de trabajo en 2013 [13]. Según datos del Instituto Mexicano del Seguro Social [14], en los "riesgos laborales por actividad económica", para 2013 tenemos a la "Construcción de edificaciones y obra de ingeniería civil" como la segunda más riesgosa con el $7.8 \%$ de los casos, además, en "días subsidiados por grupo de actividad económica", se lleva el primer lugar con el $9.1 \%$ de los casos, así como en "defunciones", con el 19.8\%.

Considerar que existe una relación que por cada accidente que ocurre, podrían identificarse alrededor de 300 incidentes previos, nos indica que la investigación sistemática de incidentes puede ser un método de prevención de accidentes [15].

Como parte de la evolución del proceso constructivo actual hacia uno más sustentable $y$, enfocado en cuidar de la salud de todos los involucrados en el proyecto de construcción, surge la necesidad de contar con un modelo mediante el cual sea posible determinar el grado de buena gestión de un proyecto de construcción de viviendas, integrando los aspectos medioambiental y de seguridad ocupacional.

\section{OBJETIVO}

El objetivo de este trabajo fue desarrollar un modelo para evaluar la gestión de la sustentabilidad y seguridad de proyectos de ingeniería civil, en particular proyectos de 
vivienda hechos en México y que, a su vez, esta evaluación sea una guía de gestión para proyectos posteriores. Las características del modelo son las siguientes:

- Que sea de fácil aplicación.

- Que contenga una lista de verificación con parámetros de ambos enfoques de gestión.

- Que establezca un nivel de relevancia para cada criterio.

- Que tenga índices de gestión con los que se pueda evaluar y muestren el nivel de éxito de la gestión.

- Que los criterios y parámetros sirvan de pauta para la mejora en futuros proyectos.

Con estos objetivos se pretende brindar beneficios tales como conocer los parámetros que se consideran más relevantes $y$, por ende, en los que hay que prestar atención extra a la hora de llevar a cabo un proyecto y también conocer el grado de éxito en la gestión del proyecto en materia de sustentabilidad y seguridad.

\section{METODOLOGÍA}

Para alcanzar los objetivos señalados en el apartado anterior se siguen los pasos que se enlistan a continuación:

* Primeramente, se realiza una revisión bibliográfica en la que se identifican los principales parámetros comúnmente utilizados en materia de sustentabilidad y seguridad de construcciones. Posteriormente, se seleccionan los que se consideran útiles para los fines de este proyecto.

* Una vez seleccionados los parámetros, se agrupan los que así lo permiten y se denominan criterios.

* Se crea una lista de verificación ordenada de todos los criterios y, por medio de la opinión de un grupo de expertos, se valida la importancia de cada criterio para conocer su relevancia y, según el caso, saber cuáles deben desecharse. Para esto se utiliza el método Delphi.

* Se hacen los ajustes pertinentes a la lista de verificación tomando en cuenta la opinión del grupo de expertos $y$, después, se identifica en qué etapa del ciclo de vida del proyecto se debe verificar cada uno de estos criterios.

* Se crea y revisa el modelo de gestión para el uso de los criterios propuestos.

- Se aplica el modelo, se identifican imperfecciones y se corrigen.

* Se realiza la revisión final del modelo de gestión.

* Se aplica de nuevo, se obtienen conclusiones acerca del desempeño y se emiten recomendaciones para los administradores de los proyectos de los casos de aplicación.

\section{EL MODELO}

Después de la selección, depuración, evaluación y recomendaciones hechas por los expertos, el modelo quedó reducido a treinta criterios, de los cuales veinte corresponden a Construcción sustentable y diez a la
Gestión de la seguridad. Fueron distribuidos de la siguiente manera:

\section{Criterios de Construcción sustentable:}

1. Naturaleza de los materiales.

2. Durabilidad de los materiales.

3. Utilización de materiales y recursos recuperados. Reutilización de materiales.

4. Reciclaje de materiales.

5. Materiales con distintivos de garantía de calidad ambiental.

6. Formación y profesionalización de todos los trabajadores en el ámbito ambiental.

7. Energía consumida en el transporte.

8. Energía utilizada en el proceso de construcción de la vivienda.

9. Consumo energético de la vivienda.

10. Utilización de fuentes de energía naturales mediante dispositivos tecnológicos.

11. Residuos y emisiones generados en el proceso de construcción de la vivienda.

12. Residuos y emisiones generados durante la actividad de la vivienda.

13. Residuos y emisiones generados en la deconstrucción de la vivienda.

14. Control de la contaminación acústica.

15. Diseño flexible de la vivienda.

16. Guía para los usuarios en la operación eficiente de la vivienda.

17. Calidad de vida de los habitantes.

18. Instalaciones en la vivienda que ahorran agua.

19. Valor ecológico del suelo y cuidado de los alrededores.

20. Urbanización sustentable (estacionamiento, drenaje, etcétera).

\section{Criterios de Gestión de la seguridad:}

1. Planear seguridad desde el diseño.

2. Documentar accidentes y daños.

3. Documentar acciones preventivas.

4. Documentar fallas de maquinaria y equipo.

5. Capacitar al personal en materia preventiva.

6. Documentar entrenamiento de personal.

7. Conocer el origen de los accidentes.

8. Realizar el análisis de riesgos.

9. Sistema de control y prevención de riesgos.

10. Informar al personal de resultados del programa.

\section{CASOS DE APLICACIÓN}

El modelo se prueba en dos proyectos: uno en la ciudad de Guadalajara, Jalisco y otro en la ciudad de Monterrey, Nuevo León. Se trata de que sean proyectos diferentes para ver cómo se comporta el modelo en cada situación.

La primera aplicación del modelo es un proyecto de construcción de viviendas de tipo residencial medio, en un desarrollo urbano de dos mil viviendas. Este desarrollo 
consta de siete tipos diferentes de viviendas y se encuentra en la ciudad de Guadalajara, en el municipio de Zapopan, estado de Jalisco, México.

El costo aproximado de una de estas viviendas es de $\$ 900,000.00$ pesos. Estas casas son de dos niveles y tienen tres recámaras, cuentan con cocina, sala, comedor, estancia, estacionamiento y dos y medio baños. Son 95 metros cuadrados de construcción en un terreno de 99 metros cuadrados.

Los resultados obtenidos de la aplicación del modelo en este proyecto son los siguientes: de construcción sustentable se completó el $46.19 \%$ de los criterios y de seguridad el $50.07 \%$. En las figuras 1 y 2 , se muestran en color rojo la puntuación obtenida en cada uno de los criterios, junto a la puntuación máxima posible en color azul. El espacio entre ambas líneas es la oportunidad de mejora del criterio.

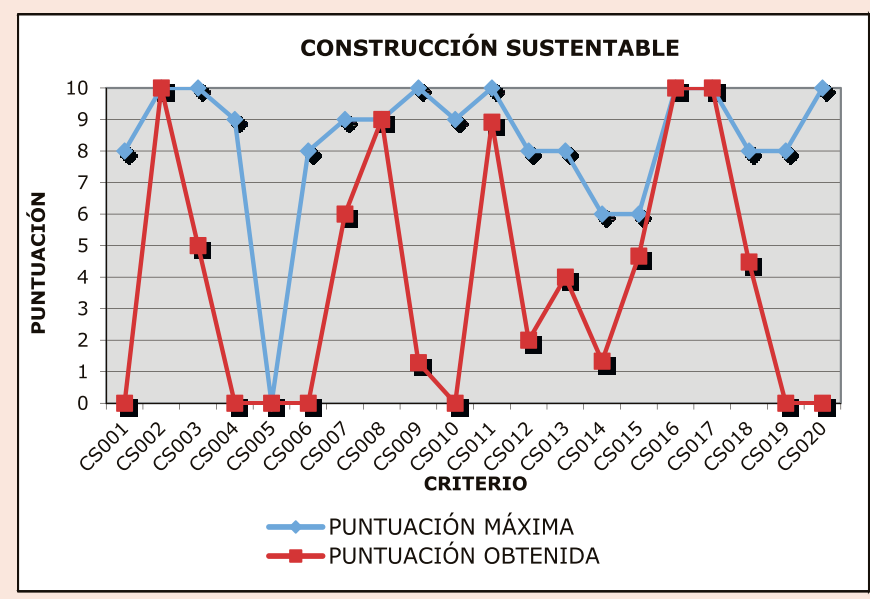

Figura 1. Análisis de resultados de Construcción sustentable.

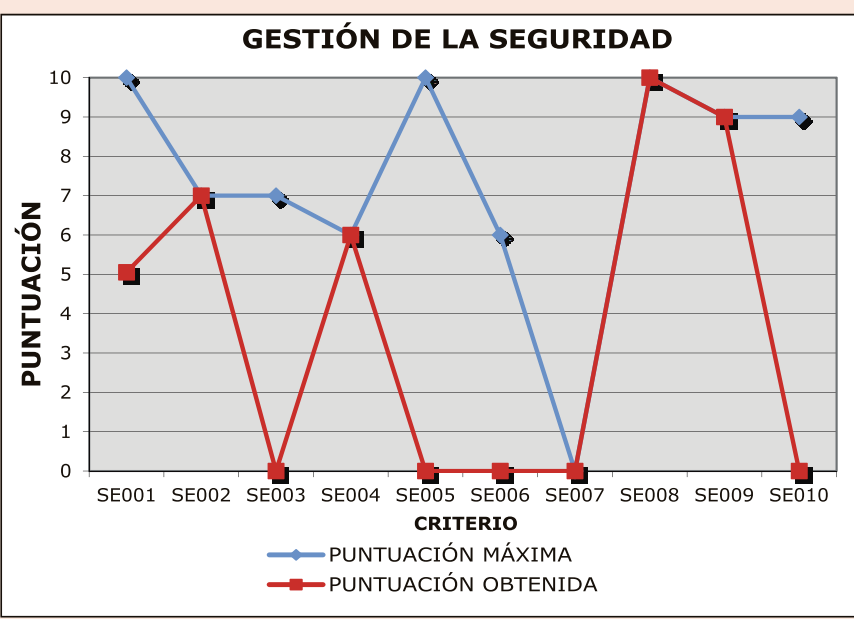

Figura 2. Análisis de resultados de Gestión de la seguridad.

La segunda aplicación del modelo es en un proyecto de construcción de viviendas residenciales, en un desarrollo urbano que se localiza al sur de la ciudad de Monterrey, en el estado de Nuevo León, México.

Las características generales de estas viviendas son un terreno de 350 metros cuadrados, construcción de 295 metros cuadrados, tres recámaras (la principal con baño vestidor), sala-comedor, cocina-antecomedor, cuarto de lavado cerrado, cuatro y medio baños, cochera doble, amplio jardín y cuarto de servicio.

En este caso, los resultados que se obtuvieron fueron que se completó el $28.83 \%$ de los criterios de construcción sustentable y el $72.06 \%$ de gestión de la seguridad. A continuación se presentan las figuras 3 y 4 correspondientes a este proyecto.

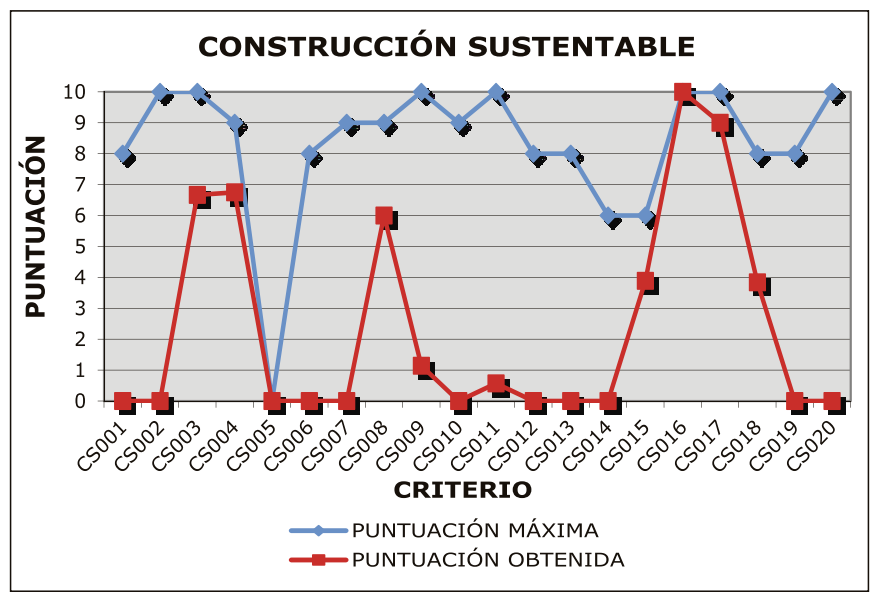

Figura 3. Análisis de resultados de Construcción sustentable.

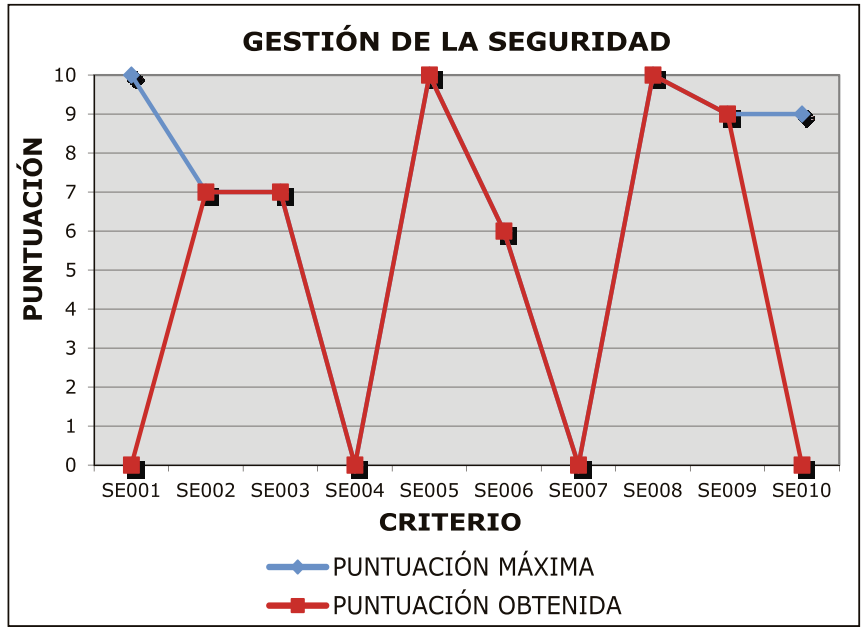

Figura 4. Análisis de resultados de Gestión de la seguridad.

\section{CONCLUSIONES}

Mediante el uso de este modelo, se logró determinar, con una buena aproximación a la realidad de cada proyecto y por medio de una calificación que se obtiene con la suma de los puntajes obtenidos en los criterios, el buen grado de gestión desde el punto de vista de cada uno de los enfoques. 
En relación a la sustentabilidad de las construcciones, la percepción en la industria sigue siendo que la construcción sustentable implica un sobrecosto, que a pesar de que se han modificado algunas normas como las de ahorro energético, y se han otorgado incentivos para empresas inmobiliarias y para usuarios orientados al ahorro energético y ahorro en el consumo de agua, el usuario final no está dispuesto a pagar el costo adicional, que es solamente un incremento en la inversión inicial.

Así también, la mayoría de proyectos que tienen enfoques sustentables se limitan a propiedades térmicas como cierto aislamiento, a la instalación de dispositivos, electrodomésticos y luminarias de bajo consumo, llaves ahorradoras de agua, etcétera, lo que no demuestra un verdadero compromiso con el medio ambiente; por otro lado hay quienes se esfuerzan un poco más e incorporan en sus proyectos algunos aspectos de diseño bioclimático.

En cuanto a la gestión de la seguridad, la práctica cotidiana se basa en que se cumpla con el pago de las cuotas o tasas, o sea, ofrece un seguro en caso de ocurrir un accidente laboral, pero no se preocupa por ir a la raíz del problema ni por verificar la aplicación de mecanismos para la prevención de riesgos. Es decir, los constructores consideran que implementar medidas de seguridad y salud es un pago extra, y por eso se limitan a las condiciones más básicas como señalizaciones, protección general de la obra $y$ algunas protecciones personales.

\section{BIBLIOGRAFÍA}

[1] Iniciativa para Edificios Sostenibles y Clima (UNEP$S B C l)$. Promoviendo políticas y prácticas para edificios y construcciones N.d: Programa de las Naciones Unidas para el Medio Ambiente, [En línea] Disponible: http://www.unep. org/sbci/pdfs/sbci_2pager_spanish.pdf.

[2] G. Insunza y C. R. Dávila. "Desafíos de los programas de vivienda sustentable en México" Cuadernos de vivienda y urbanismo, vol. 4, no. 7, pp. 60-74, Enero-Junio 2011.

[3] R. Kaineg et al. (2013) NAMA Apoyada para la Vivienda Sustentable en México - Acciones de Mitigación y Paquetes financiero. [En línea] Disponible: <http://www.conavi.gob. $\mathrm{mx} /$ images/documentos/sustentabilidad/NAMA_mexicana_ de_vivienda_sustentable_documento_espa $\% \mathrm{C} 3 \% \mathrm{~B} 1$ ol.docx $>$

[4] Cámara Mexicana de la Industria de la Construcción (2014). Plan de manejo de residuos de la construcción y la demolición. [En línea] Disponible: <http://www.fic.org.mx/ OTTIC/CMIC/PMrcdCompleto.pdf>

[5] L. Garrido. Análisis de proyectos de arquitectura sotenible: Naturalezas artificiales (2001-2008). España: McGraw Hill, 2008.

[6] Diario oficial de la federación (2014). Programa Nacional de Vivienda 2014-2018. [En línea] Disponible: <http:// www.dof.gob.mx/nota_detalle.php?codigo $=5342865 \& \mathrm{fec}$ ha $=30 / 04 / 2014>$

[7] E. León, (2008). Guía de agua y construcción sustentable. [En línea] Disponible: <http://www.agua.org.mx/ h2o/index.php?option $=$ com_docman\&task $=\mathrm{doc}_{-}$ download\&gid=252\&ltemid $=100162>$

[8] "Guía CONAVI criterios e indicadores para desarrollos habitacionales sustentables". México: CONAVI, 2008.

[9] E. Rocha, "Construcciones sostenibles: materiales, certificaciones y LCA", Revista nodo. Vol. 11, no. 6, pp. 99-116, Julio-Diciembre 2011.
[10] M. Umaña, "Sostenibilidad y ciclo de vida de los materiales," M.S. tesina, Universidad Politécnica de Cataluña, Barcelona, España, 2011.

[11] R. Solís, C. Alcudia y C. Campos. "Seguridad y salud en la construcción masiva de vivienda en México: caso de estudio" Ingeniería y Universidad. Vol. 10, no. 2, pp. 209-222, JulioDiciembre 2006.

[12] A. López. "La construcción: uno de los trabajos con mayores riesgos laborales en el mundo", Revista de divulgación científica CienciAcierta digital. Vol. 9, no. 33, Enero-Marzo 2013.

[13] Commonly Used Statistics (2015). [En línea] Disponible: Occupational Safety \& Health Administration <https://www. osha.gov/oshstats/commonstats.html>

[14] IMSS (2014). Información sobre Accidentes y Enfermedades de Trabajo Nacional 2004-2013. [En línea] Disponible: <http://www.stps.gob.mx/bp/secciones/dgsst/estadisticas/ Nacional\%202004-2013.pdf>

[15] Secretaría de Previsión y Trabajo. Prácticas Seguras en la Industria de la Construcción. [En línea] Disponible: <http://www.stps.gob.mx/bp/secciones/dgsst/ publicaciones/prac_seg/construccion/PA-06.pdf>

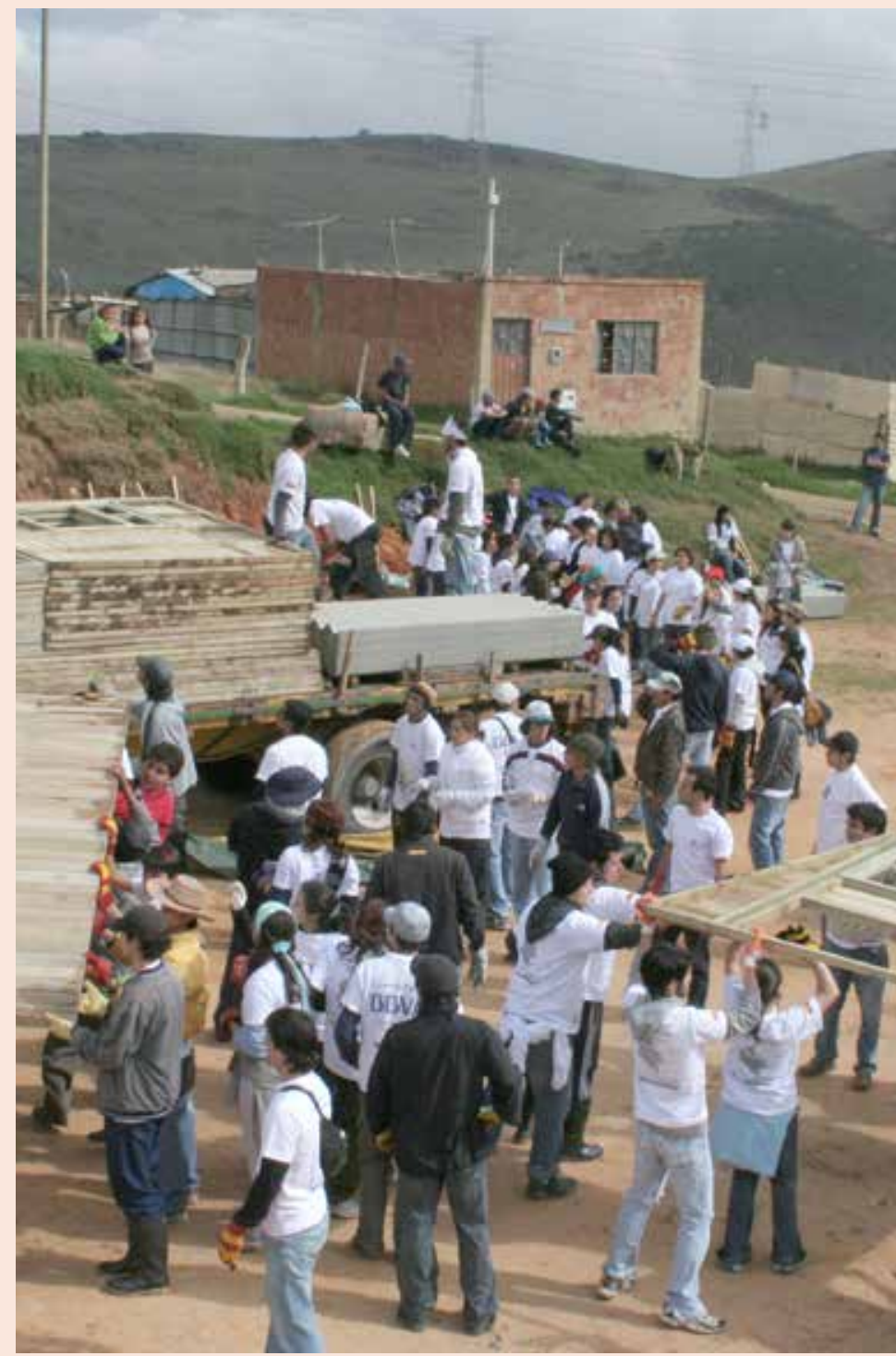

